\title{
Single-Molecule Dynamics of Lysozyme Processing Distinguishes Linear and Cross-Linked Peptidoglycan Substrates
}

\author{
Yongki Choi, ${ }^{\dagger} \ddagger$ Issa S. Moody, ${ }^{\S}$ Patrick C. Sims, ${ }^{\ddagger}$ Steven R. Hunt, ${ }^{\ddagger}$ Brad L. Corso ${ }^{\ddagger}$ David E. Seitz, ${ }^{\perp}$ \\ Larry C. Blaszcazk, ${ }^{\perp}$ Philip G. Collins, ${ }^{*},+, \neq$ and Gregory A. Weiss ${ }^{*}, \S, \|$
}

${ }^{\dagger}$ Institute for Surface and Interface Science and Departments of ${ }^{\ddagger}$ Physics and Astronomy, ${ }^{\S}$ Molecular Biology and Biochemistry, and "Chemistry, University of California, Irvine, California 92697, United States

${ }^{\perp}$ Muroplex Therapeutics, Inc., 351 West 10th Street \#355, Indianapolis, Indiana 46202, United States

Supporting Information

ABSTRACT: The dynamic processivity of individual T4 lysozyme molecules was monitored in the presence of either linear or cross-linked peptidoglycan substrates. Single-molecule monitoring was accomplished using a novel electronic technique in which lysozyme molecules were tethered to single-walled carbon nanotube field-effect transistors through pyrene linker molecules. The substratedriven hinge-bending motions of lysozyme induced dynamic electronic signals in the underlying transistor, allowing long-term monitoring of the same molecule without the limitations of optical quenching or bleaching. For both substrates, lysozyme exhibited processive low turnover rates of $20-50 \mathrm{~s}^{-1}$ and rapid (200-400 s ) nonproductive motions. The latter nonproductive binding events occupied $43 \%$ of the enzyme's time in the presence of the cross-linked peptidoglycan but only $7 \%$ with the linear substrate. Furthermore, lysozyme catalyzed the hydrolysis of glycosidic bonds to the end of the linear substrate but appeared to sidestep the peptide cross-links to zigzag through the wild-type substrate.

$\mathrm{T}$ he key roles contributed to biological processes by enzymes make correlating enzyme motions with their catalytic functions an important and challenging problem., The spatial and temporal heterogeneity of enzymes in bulk solution prevents ensemble measurements from examining an enzyme's conformational dynamics along its reaction coordinates. $^{3-5}$ However, single-molecule studies, typically using fluorescence resonance energy transfer (FRET), can characterize the conformational dynamics ${ }^{6,7}$ and also reveal the static and dynamic disorders inherent in enzyme activities. ${ }^{2,8}$

Lysozyme is a particularly good model protein for elucidating detailed enzyme dynamics and conformational motions from single-molecule observations. ${ }^{9}$ An antibiotic component of innate immunity, lysozyme digests the peptidoglycan of bacterial cell walls. The enzyme catalyzes the hydrolysis of the glycosidic bonds connecting the repeating subunits of the cell walls between $\mathrm{N}$-acetylglucosamine (NAG) and $\mathrm{N}$ acetylmuramic acid (NAM).

During catalysis, lysozyme undergoes $8 \AA$ hinge bending motions ${ }^{10}$ that, with the addition of fluorescent labels, enable single-molecule FRET experiments. ${ }^{3}$ As visualized by FRET on bacterial surfaces, lysozyme adheres to the peptidoglycan for long periods of time. ${ }^{3}$ Such observations suggest, but do not prove, that lysozyme processively catalyzes the hydrolysis of a large number of glycosidic bonds before dissociation. FRET also proves that lysozyme interrupts its catalytic glycosidic hydrolysis with periods of rapid movements that do not result in bond hydrolysis. ${ }^{11}$ However, the degree of processivity, the reason for the rapid nonproductive motions, and the effects of substrate cross-linking remain incompletely understood for two main reasons. First, the peptidoglycan is highly heterogeneous in size and features a heavily cross-linked structure involving connections of the NAM subunits of the polysaccharide chains by pentapeptides. ${ }^{3,12}$ Second, FRET and other optical techniques are limited by fluorophore bleaching, which prevents long-term measurement of the same individual molecule. Such considerations motivate the development of new methods for examining single proteins.

To address these issues, two developments are reported here. First, we synthesized a linear peptidoglycan substrate for lysozyme that includes non-cross-linked tripeptides (Figure 1a). Second, we developed nanocircuits comprising individual lysozyme molecules attached to single-walled carbon nanotube (SWNT) field-effect transistors (FETs). The dynamic motions of the attached lysozyme induce fluctuations in the SWNT-FET conductance through a charge gating effect, similar to previous work with SWNT-FET sensors. ${ }^{13-15}$ This electronic rather than optical transduction allows monitoring of the dynamic interactions of individual lysozyme molecules over long periods of time. Combining these two advances has provided new insights into lysozyme processing of peptidoglycans.

To examine the dynamics of lysozyme, individual lysozyme molecules were attached to the sidewalls of SWNT-FETs, as shown in Figure 1b. Atomic force microscopy (AFM) images confirmed the attachment of one lysozyme within a window opened by the electron beam in an insulating poly(methyl methacrylate) (PMMA) layer placed over the entire device (Figure 1c). ${ }^{16}$ An S90C variant of pseudo-wild-type T4 lysozyme $^{17}$ (used throughout the work reported here) provided a single thiol for conjugation to a pyrene-linked maleimide. ${ }^{13,18}$ The aromatic pyrene of this linker can strongly adhere to the SWNT sidewall via $\pi-\pi$ interactions. ${ }^{13,19}$ Multiple washing

Received: December 9, 2011

Published: January 9, 2012 
(a)

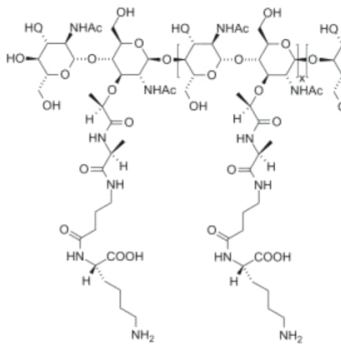

(b)

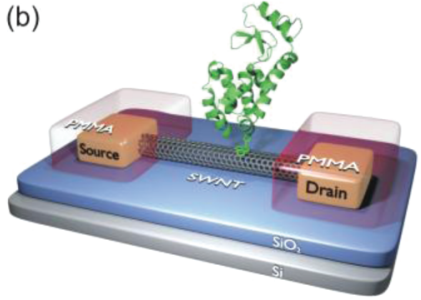

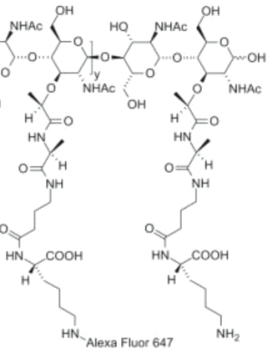

(c)

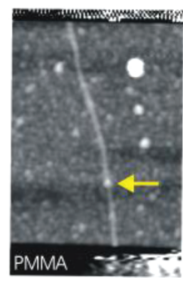

Figure 1. (a) Chemical structure of the synthesized peptidoglycan substrate. (b) Schematic diagram of a single-molecule T4 lysozyme SWNT-FET circuit. (c) AFM topography of a SWNT-FET after coating with the pyrene linker, lysozyme incubation, and washing to reduce nonspecific binding. The arrow indicates a site of lysozyme attachment.

steps were then used to tailor the density of the lysozyme attachments to yield an average of one attachment per device.

As the enzyme moves, charged surface functionalities near the SWNT attachment site can modulate the source-drain conductance $G(t)$ of the underlying device. Electrical measurements were performed with the device submerged in electrolyte (phosphate-buffered saline, $\mathrm{pH}$ 7.5) under an applied sourcedrain voltage of $0.1 \mathrm{~V}$ and an electrolyte-drain bias of $0 \mathrm{~V}$ (controlled by a Pt reference electrode). Devices were exposed to excess substrate and measured for $600 \mathrm{~s}$; the same device was then thoroughly rinsed with water to remove surface-bound substrate before being probed a second time with a different substrate. The two substrates were tested in alternating orders on different devices to protect against systematic bias. Our analysis filtered the DC and lowest frequency $(<10 \mathrm{~Hz}) \mathrm{AC}$ components of $G(t)$ in order to focus on time-varying fluctuations and transients of $\Delta G(t)$.

Three distinct behaviors were observed and are categorized in Figure 2. The most common behavior was two-state $G(t)$ fluctuation switching asymmetrically at an average rate of 30.0 $\mathrm{s}^{-1}$ (Figure 2a, colored green). A second behavior involved fluctuations between the same two $G(t)$ levels at the much higher average rate of $287 \mathrm{~s}^{-1}$ (Figure $2 \mathrm{~b}$, blue). A relatively featureless, inactive behavior also occurred (Figure 2c, black), though this was primarily observed when measurements were performed in the absence of the lysozyme substrate peptidoglycan. Previous FRET measurements have proven that lysozyme motions are absent or disorganized in the absence of substrate but that, when present, substrate can drive one-dimensional hinge bending motions at the same two rates we observed. ${ }^{11}$ The lower rate is understood to be caused by productive processing of substrate by the enzyme, while the higher rate is associated with nonproductive, catalytically ineffective motions of the lysozyme. ${ }^{11}$

Long-duration measurements on single lysozyme molecules are possible with the SWNT-FET architecture. Five example measurements are shown in Figure 3 with the data points colored to correspond to the three behaviors defined above.

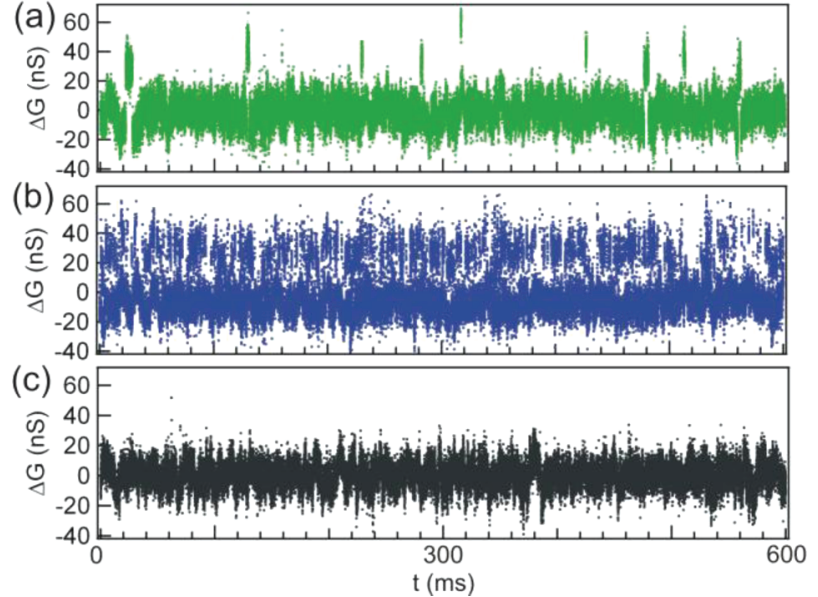

Figure 2. Source-drain conductance fluctuations $\Delta G(t)$ of a lysozyme device in the presence of the cross-linked substrate. The colors differentiate the three types of observed signal behavior, which included (a) slow switching with catalytic turnover (green), (b) rapid nonproductive switching (blue), and (c) inactivity (black).

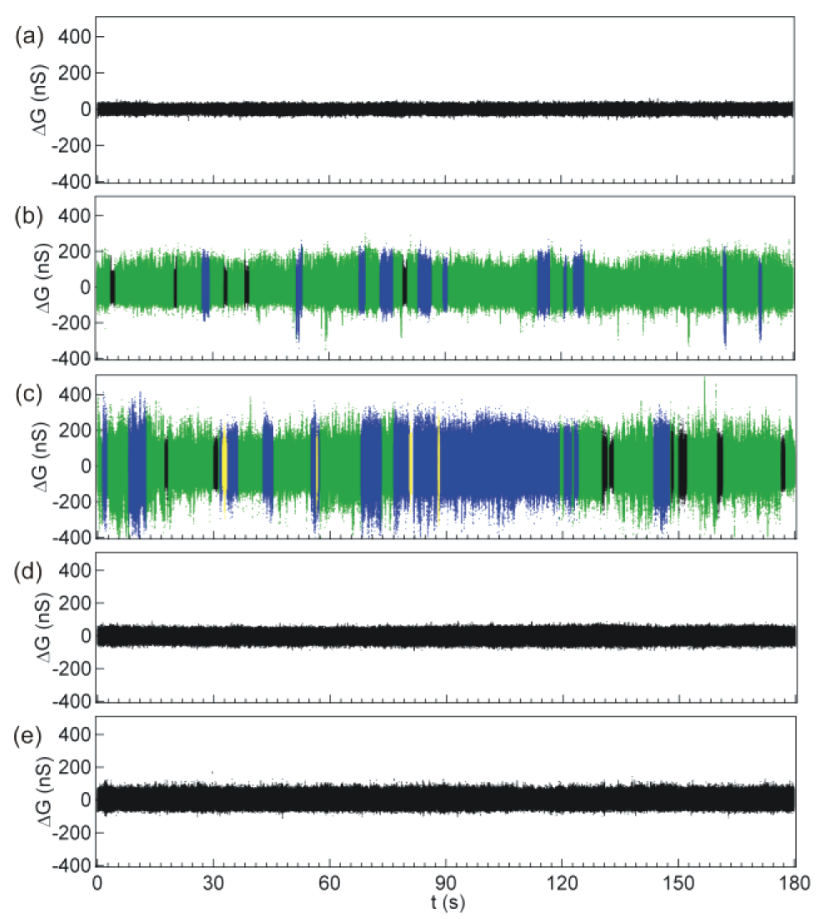

Figure 3. Long-duration source-drain conductance fluctuations. (a) In the absence of substrate, no conductance fluctuations were observed, as demonstrated here for a single lysozyme device incubated in phosphate buffer. Addition of the (b) linear or (c) cross-linked substrate resulted in the switching described in the text. Control experiments using the (d) E11H or (e) T26E catalytically inactive T4 lysozyme variant showed no activity when probed with the crosslinked (shown) or linear (not shown) substrate.

Figure $3 a-c$ was obtained from a single lysozyme device measured either without substrate (Figure 3a) or with one of two different substrates described in more detail below. Figure 3d,e shows control measurements from devices incorporating two catalytically inactive lysozyme variants, ${ }^{20} \mathrm{~T} 26 \mathrm{E}$ and $\mathrm{E} 11 \mathrm{H}$. The two mutated residues, Thr and Glu, play key roles in the lysozyme mechanism for the catalysis of glycoside hydrolysis. The T26E variant produces a covalent adduct with the 
peptidoglycan substrate and thus provides a constitutively substrate-bound version of the lysozyme. The E11H variant is also catalytically inoperable but does not form a covalent bond to the substrate. The absence of two-level fluctuations when substrate was absent (Figure 3a) or when the two control variants were probed with substrate (Figure 3d,e) confirmed that the two-level $\Delta G(t)$ dynamics was caused by substratelysozyme interactions and catalysis and not merely the presence of one substrate or the other.

The main focus of this report is to compare lysozyme processing of two different peptidoglycan substrates, one linear and the other cross-linked. The linear lysozyme substrate was obtained through chemical synthesis as described in the Supporting Information (SI). Designed to mimic bacterial cell walls, the linear substrate featured an extended (NAG-NAM) polysaccharide. Appended to each NAM subunit were tripeptides that were linked to lactic acid but did not crosslink polysaccharide fragments (Figure 1a). Alexa Fluor 647 dye was incorporated at a density of $\sim 5 \%$ on the peptides; the fluorophore was not used in the present experiments and would not be expected to alter the enzyme dynamics. Commercially obtained cell walls of peptidoglycan from Micrococcus luteus provided a second substrate for an examination of the effects of substrate cross-linking on the enzyme catalysis. As shown by fluorescence-based assays with ensemble or bulk enzyme, T4 lysozyme could hydrolyze both substrates (Figures S3 and S4 in the SI).

Figure $3 b, c$ shows a comparison of the catalytic activities of lysozyme processing of linear and cross-linked substrates, respectively. Both substrates allowed processive catalysis by lysozyme (green segments) but exhibited different proportions of the nonproductive (blue) and inactive (black) behaviors. T4 lysozyme does not require, nor is it hindered by, the peptide cross-links of the substrate for its processive movement. Nevertheless, the linear substrate fundamentally altered the distribution of the lysozyme dynamics. Figure 4 summarizes the

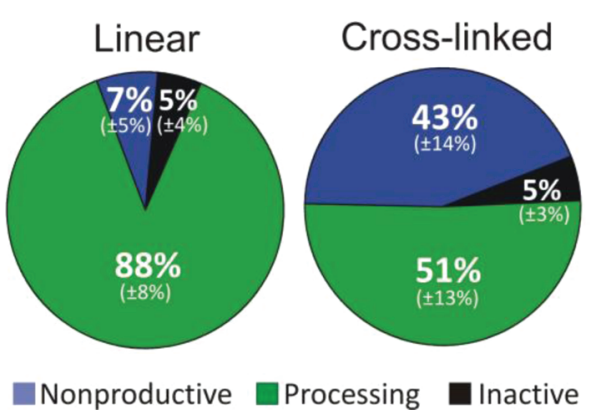

Figure 4. Percentages of lysozyme activities in the presence of the (left) linear or (right) cross-linked peptidoglycan substrate. The standard deviations for the activities are indicated in parentheses $(n=$ 6 for the linear substrate and $n=8$ for the cross-linked substrate).

analysis of 24 independent measurements performed using 14 different devices, of which Figure 3 is representative. On average, lysozyme spent $88 \%$ of the total time processing the linear substrate and made few rapid, nonproductive motions ( $7 \%$ of its time).

This processing of linear substrate contrasts sharply with observations made in the presence of the cross-linked substrate. The lysozyme increased the amount of time spent in rapid, nonproductive motions from 7 to $43 \%$ in the presence of the cross-linked peptidoglycan substrate (Figure 4). This difference in activity was very reproducible, regardless of whether the cross-linked or linear substrate was tested first. Furthermore, no degradation of lysozyme activity was observed during the reported experiments. Monitoring of serial processing the two different types of substrates by the same lysozyme molecule proved that the peptide cross-links trap the enzyme in this rapidly oscillating, nonproductive state. We hypothesize that lysozyme catalysis stalls before attempting to transit a cross-link to reach a neighboring polysaccharide. Thus, lysozyme could zigzag across the cell wall as it processively catalyzes glycoside hydrolysis and passes across peptide cross-links.

Despite the major differences in the distribution of enzyme activities, the two substrates had chemically identical glycosidic bonds and resulted in remarkably similar kinetic rates. The fluctuation in the rates of catalytic processing (Figure 2a) and nonproductive motion (Figure $2 \mathrm{~b}$ ) changed only slightly, if at all, when one substrate was substituted for another. Ambiguity in this comparison was caused by the static disorder of individual enzymes and the local environment of the enzyme tethered to the SWNT-FETs, both of which contributed to broad rate distributions. For example, the instantaneous singlemolecule rates of catalytic turnover with the cross-linked substrate ranged from 17 to $59 \mathrm{~s}^{-1}$. Nevertheless, quantitative comparisons were made by averaging the mean rates (reported here with one standard deviation) from $100 \mathrm{~s}$ segments across multiple devices. Accordingly, the rate of catalytic turnover decreased $16 \%$, from $35.9 \pm 17.6 \mathrm{~s}^{-1}(n=9)$ for the linear substrate to $30.0 \pm 14.5 \mathrm{~s}^{-1}(n=15)$ for the cross-linked substrate. The rate of nonproductive motions decreased $13 \%$, from $329 \pm 167 \mathrm{~s}^{-1}$ for the linear substrate to $287 \pm 184 \mathrm{~s}^{-1}$ for the cross-linked substrate. Although the two substrates had overlapping ranges, the cross-linked substrate did appear to result in slightly lower rates. As might be expected, the presence of cross-links appears to slow the catalytic processing. The additional slowing of the nonproductive motions is consistent with a model in which the enzyme transits from one polysaccharide to another at cross-linked points.

The linear and cross-linked peptidoglycans exhibited similar percentages of inactive time (black data in Figures 3 and 4). During these inactive periods, which had an average duration of $1.06 \mathrm{~s}$, no switching by the lysozyme device was observed, and $G(t)$ remained in its low state. This low $G(t)$ state was identical to the value observed when no substrate was present (Figure S5b,d). Furthermore, the inactive periods occurred exclusively during periods of catalytic processing (green) and were never observed during the putative enzyme transits at peptide crosslinks (blue). These observations suggest that the inactive period is caused by dissociation that occurs when lysozyme processes to the end of a poysaccharide substrate. In this interpretation, lysozyme proceeds along the polysaccharide backbone, catalyzing the hydrolysis of multiple glycosidic bonds before dissociation occurs. Upon association of new substrate, the inactive period concludes. We observed that the enzyme always returned to a substrate-processing state (green).

Finally, we note a fourth type of behavior that was observed only with the cross-linked substrate and is represented by the yellow color in Figure 3c. These segments correspond to novel behavior in which rapid, nonproductive motion at the peptide cross-links is interrupted for $0.5-3.0 \mathrm{~s}$ with $G(t)$ stuck at its high value. Whereas the inactive periods discussed above are associated with dissociation events because of their low $G(t)$, these pauses at high $G(t)$ imply that the enzyme is stuck in an enzyme-closed, substrate-bound configuration (Figure S5c,e). 
This state occurs randomly and with a low probability, and it appears to be independent of the return to catalytic processing.

The results presented here demonstrate the tremendous potential of molecular electronics to uncover fundamental knowledge in biophysics. Lysozyme orthologues have been studied for over a century, yet the nanocircuits reported here have unveiled new information about T4 lysozyme's activities and dynamics, including its processivity and potential ability to transit peptide cross-links. Lysozyme proceeds linearly to the ends of the linear substrate but could sidestep over peptide cross-links of the cross-linked substrate. This study providing a relationship between lysozyme dynamics and function will allow deeper understanding of the precise lysozyme motions for digesting the bacterial cell wall and could guide the design of new enzymes for antibacterial and other applications.

\section{ASSOCIATED CONTENT}

\section{S Supporting Information}

Additional materials and methods, bulk fluorescence-based assay measurement of substrates, analysis of inactive enzyme periods, and power spectral density analysis. This material is available free of charge via the Internet at http://pubs.acs.org.

\section{AUTHOR INFORMATION}

\section{Corresponding Author}

collinsp@uci.edu; gweiss@uci.edu

\section{ACKNOWLEDGMENTS}

We thank the following contributors from Eli Lilly and Company (Indianapolis, IN): Elizabeth Dingess-Hammond and Brian Moser for assistance with the synthesis; Melissa Clague and Yvonne Vandenburg for the kinetic analysis of bulk T4 lysozyme hydrolysis of synthetic peptidoglycan. This work was supported financially by NIH NCI (R01 CA133592-01) and NSF (DMR-1104629 and the Center for Chemical Innovation on Chemistry at the Space-Time Limit, CHE0802913).

\section{REFERENCES}

(1) Bhabha, G.; Lee, J.; Ekiert, D. C.; Gam, J.; Wilson, I. A.; Dyson, H. J.; Benkovic, S. J.; Wright, P. E. Science 2011, 332, 234.

(2) Lu, H. P.; Xun, L. Y.; Xie, X. S. Science 1998, 282, 1877.

(3) Hu, D. H.; Lu, H. P. Biophys. J. 2004, 87, 656.

(4) Eisenmesser, E. Z.; Bosco, D. A.; Akke, M.; Kern, D. Science 2002, 295, 1520.

(5) Engelkamp, H.; Hatzakis, N. S.; Hofkens, J.; De Schryver, F. C.; Nolte, R. J. M.; Rowan, A. E. Chem. Commun. 2006, 935.

(6) Roy, R.; Hohng, S.; Ha, T. Nat. Methods 2008, 5, 507.

(7) Ha, T.; Ting, A. Y.; Liang, J.; Caldwell, W. B.; Deniz, A. A.; Chemla, D. S.; Schultz, P. G.; Weiss, S. Proc. Natl. Acad. Sci. U.S.A. 1999, 96, 893.

(8) Min, W.; English, B. P.; Luo, G. B.; Cherayil, B. J.; Kou, S. C.; Xie, X. S. Acc. Chem. Res. 2005, 38, 923.

(9) Lu, H. P. Curr. Pharm. Biotechnol. 2004, 5, 261.

(10) McHaourab, H. S.; Oh, K. J.; Fang, C. J.; Hubbell, W. L. Biochemistry 1997, 36, 307.

(11) Chen, Y.; Hu, D. H.; Vorpagel, E. R.; Lu, H. P. J. Phys. Chem. B 2003, 107, 7947.

(12) Meroueh, S. O.; Bencze, K. Z.; Hesek, D.; Lee, M.; Fisher, J. F.; Stemmler, T. L.; Mobashery, S. Proc. Natl. Acad. Sci. U.S.A. 2006, 103, 4404.

(13) Li, C.; Curreli, M.; Lin, H.; Lei, B.; Ishikawa, F. N.; Datar, R.; Cote, R. J.; Thompson, M. E.; Zhou, C. J. Am. Chem. Soc. 2005, 127, 12484 .
(14) Besteman, K.; Lee, J.-O.; Wiertz, F. G. M.; Heering, H. A.; Dekker, C. Nano Lett. 2003, 3, 727.

(15) Chen, R. J.; Bangsaruntip, S.; Drouvalakis, K. A.; Kam, N. W. S.; Shim, M.; Li, Y. M.; Kim, W.; Utz, P. J.; Dai, H. J. Proc. Natl. Acad. Sci. U.S.A. 2003, 100, 4984.

(16) Choi, Y.; Moody, I. S.; Sims, P. C.; Hunt, S. R.; Corso, B. L.; Perez, I.; Weiss, G. A.; Collins, P. G. Science 2012, 335, 319.

(17) Matsumura, M.; Matthews, B. W. Science 1989, 243, 792.

(18) Chen, R. J.; Zhang, Y.; Wang, D.; Dai, H. J. Am. Chem. Soc 2001, $123,3838$.

(19) Hermanson, G. T. Bioconjugate Techniques, 2nd ed.; Academic Press: San Diego, 2008.

(20) Kuroki, R.; Weaver, L. H.; Matthews, B. W. Proc. Natl. Acad. Sci. U.S.A. 1999, 96, 8949. 